\section{§24. Plasma Instabilities and Anomalous Resistivity in the Current Sheet}

\author{
M oritaka, T. (N agoya Univ.), Horiuchi, R., Ohtani, \\ $\mathrm{H}$., Ishizawa, A.
}

Magntic reconnection can lead to topological change of magnetic field and fast energy conversion from magnetic field to particles and is widely believed as a fundamental process of the energetically active phenomena observed in the solar colona, the geomagnetic tail and fusion plasma. Instead of bynary collisions, microscopic nonideal effect can dissipate magnetic field energy and cause collisionless magnetic reconnection in these systems.

A variety of microscopic plasma instabilities which can excite in the curent sheet have so far been studied by linear analyses. It is important to clarify how the excitation of these instability causes nonideal effect and collisionless magnetic reconnection. In this paper we investigate microscopic effect in collisionless magnetic reonnection by examing the force balance equation when these instabilities grow.

The simulation is carried out by using $2+1 / 2$ dimensional explicit electromagnetic particle simulation code. We adopt the Harris type equibrium with the antiparallel magnetic field configuation. Periodic and fixed boundary conditions are imposed in equibilium current direction and along the gradient of magnetic field. The force balance expressed by two-fluid equation is examined using the particle simulatin result. The relationship between nonideal effect created by microscopic plasma instabilities and the generation of electric field is investigated besed $n$ this force balance equation.

In the early phase of the development of current sheet, the electromagnetic wave is excited at the periphery of the currnt sheet. The spectrum of this wave agrees with the linear analysis of LHDI ${ }^{1)}$. Fig.1(top) shows the structure of magnetic field and the force balance at the phase of LHDI. The nonlinear coupling of the perturbations of the electic field and particle density $\tilde{n} \tilde{\mathbf{E}}$ contributes the outward flow of particle $\langle\mathbf{v}\rangle$ in the periphery of the current sheet. This outward flow result in the deformation of the equilibrium current sheet profile such as the localization of current density and magnetic flux. The change of the meandering orbit across the neutral sheet by this particle transport lead to the particle accelation by the pressure tensor term in neutral sheet. But average electric field in the neutral sheet or magnetic flux reduction are not found in this phase.

After the saturation of LHDI, the growth of the longer electromagnetic wave is observed at the

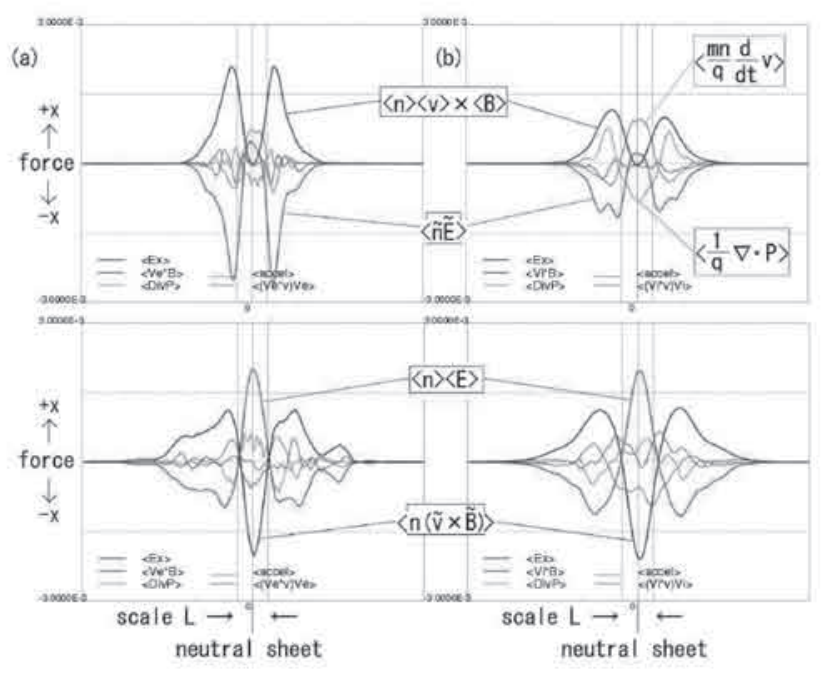

Figure 1: The spatial profile of force balance of electron (a) and ion (b) ; LHDI phase (top) and DKI phase (bottom)

neutral sheet ${ }^{2) 3)}$. This wave has good agreement with linear anlysis of $\mathrm{DKI}^{4}$. The average electric field $\langle\mathbf{E}\rangle$ appears and current density dissipates at neutral sheet. The reduction of total magnetic flux consistent with the DC electric field is found in this phase. Fig.1(bottom) shows the magnetic field and the force balance at the phase of DKI. The force balance at the neutral sheet is changed by the excitation of DKI at the center of current sheet. The fluctuations of magnetic field and current density form the finite wavy component $\left\langle\tilde{\mathbf{u}}_{j} \times \tilde{\mathbf{B}}\right\rangle$ in Lorentz force term, and this term balances the average electric field. The anomalous resistivity examined from the damping rate of equilibrium current is the same order of the effective resistivity examined from DC electric field. The interaction between the fluctuation of magnetic field and microscpic plasma flow dissipates the momentum of particles and create anomalous resistivity.

While the magnetic flux is re-distibuted in the phase of LHDI, anomalous resistivity and magnetic flux reduction are generated through the nonlinear wave-particle interaction in the DKI phase. It is concluded that the DKI play an important role in creating anomalous resistivity and triggering collisinless magnetic reconnection.

\section{References}

1) R.C.Davidson, N.T.Gladd, C.S.Wu and J.D.Huba Phys.Fluids, 20, 301(1977)

2) Z.Zhu and R.M.Winglee, J.Geophys.Res, 101, 4885(1996)

3) R.Horiuchi and T.sato, Phys.Plasmas, 6, 4565(1999)

4) W.Daughton, Phys.Plasmas, 6,1329(1999) 